\title{
Viral proteins expressed in the protozoan parasite Eimeria tenella are detected by the chicken immune system
}

\author{
Virginia Marugan-Hernandez ${ }^{1 *}$, Charlotte Cockle ${ }^{1}$, Sarah Macdonald ${ }^{1}$, Elaine Pegg ${ }^{1}$, Colin Crouch²,
} Damer P. Blake ${ }^{1}$ and Fiona M. Tomley ${ }^{1}$

\begin{abstract}
Background: Eimeria species are parasitic protozoa that cause coccidiosis, an intestinal disease commonly characterised by malabsorption, diarrhoea and haemorrhage that is particularly important in chickens. Vaccination against chicken coccidiosis is effective using wild-type or attenuated live parasite lines. The development of protocols to express foreign proteins in Eimeria species has opened up the possibility of using Eimeria live vaccines to deliver heterologous antigens and function as multivalent vaccine vectors that could protect chickens against a range of pathogens.

Results: In this study, genetic complementation was used to express immunoprotective virus antigens in Eimeria tenella. Infectious bursal disease virus (IBDV) causes Gumboro, an immunosuppressive disease that affects productivity and can interfere with the efficacy of poultry vaccination programmes. Infectious laryngotracheitis virus (ILTV) causes a highly transmissible respiratory disease for which strong cellular immunity and antibody responses are required for effective vaccination. Genes encoding the VP2 protein from a very virulent strain of IBDV (vVVP2) and glycoprotein I from ILTV (g) were cloned downstream of $5^{\prime} E t-A c t i n$ or $5^{\prime} E t-$ TIF promoter regions in plasmids that also contained a mCitrine fluorescent reporter cassette under control of the $5^{\prime} E t-M / C 1$ promoter. The plasmids were introduced by nucleofection into E. tenella sporozoites, which were then used to infect chickens. Progeny oocysts were sorted by FACS and passaged several times in vivo until the proportion of fluorescent parasites in each transgenic population reached $\sim 20 \%$ and the number of transgene copies per parasite genome decreased to $<10$. All populations were found to transcribe and express the transgene and induced the generation of low titre, transgene-specific antibodies when used to immunise chickens.
\end{abstract}

Conclusions: E. tenella can express antigens of other poultry pathogens that are successfully recognised by the chicken immune system. Nonetheless, further work has to be done in order to improve the levels of expression for its future use as a multivalent vaccine vector.

Keywords: Eimeria, Transgenic, Delivery vector, Multivalent vaccine, Poultry diseases

Abbreviations: BSA, Bovine serum albumin; CjaA, Campylobacter jejuni antigen; ConA, Concanavalin A; ELISA, Enzymelinked immunosorbent assay; EtMic1, Eimeria tenella microneme protein 1; EYFP, Enhanced yellow fluorescent protein; FACS, Fluorescence-activated cell sorting; FBS, Foetal bovine serum; gB/I/D, Virus glycoprotein B///D; HRP, Horseradish peroxidase; HVT, Herpes virus of turkey; IBDV, Infectious bursal disease virus; ILTV, Infectious laryngotracheitis virus; IMAC, Immobilized metal ion affinity chromatography; MDBK, Madin-Darby bovine kidney; PBMC, Peripheral blood mononuclear cells; qPCR, Quantitative PCR; REMI, Restriction enzyme-mediated integration; RT-PCR, Real time PCR; SDSPAGE, Sodium dodecyl sulphate polyacrylamide gel electrophoresis; TIF, Translation initiation factor; VVVP2, Very virulent viral protein 2 (capsid protein).

\footnotetext{
* Correspondence: vhernandez@rvc.ac.uk

${ }^{1}$ The Royal Veterinary College, University of London, Hawkshead Lane, North

Mymms AL9 7TA, UK

Full list of author information is available at the end of the article
} 


\section{Background}

The poultry industry provides the principal source of animal protein in many developed and developing countries [1]. The adaptability of poultry production systems has fuelled expansion of the industry to most areas of the world with high productivity and relatively low costs. However, intensive systems and backyard flocks remain threatened by viral, bacterial and parasitic infections that compromise the economics of meat and egg production, cause animal welfare problems [2] and can be a significant source of zoonotic transmission to humans [3, 4]. Control of infectious diseases that challenge poultry production is critical [5] and chickens are more intensively vaccinated than any other livestock animals. In Europe, programmes include a minimum of four vaccinations for broilers whilst layers and breeders can receive up to 20 vaccinations, many to immunise against common viral diseases including Marek's disease, Newcastle disease, Gumboro (infectious bursal disease), infectious bronchitis and infectious laryngotracheitis [6].

Coccidiosis is a disease caused by Eimeria species parasites. In chickens infection can incur a range of clinical symptoms including malabsorption, diarrhoea and haemorrhage. The parasites are transmitted by the faecal-oral route [7]. Coccidiosis control is predominantly achieved by chemoprophylaxis with ionophorous antibiotics and/or chemical anticoccidials. Vaccination with formulations of live wild-type or attenuated parasites is widely used, and very effective, in layers and breeders and with an increased role in the broiler market due to new regulations (e.g. Veterinary Feed Directive in the US) and a preference of retailers to sell meat without antibiotics or with reduced antibiotics. [8]. Protocols which allow genetic complementation of Eimeria to express heterologous protein-coding sequences [9] raises the interesting possibility that existing Eimeria vaccines could be engineered to express antigens derived from a wide range of poultry pathogens [10, 11]. Eimeria genomes are much larger than those of viral vectors, in consequence they can tolerate the insertion and expression of several foreign antigens. They induce a broad range of potent immune responses following oral administration [12] having the potential to be exploited as a flexible oral vaccine vector for intracellular and extracellular pathogens.

In this study we have expressed in Eimeria tenella specific antigens that have already been proven capable of inducing protection against two economically important viral diseases of chickens. Infectious bursal disease virus (IBDV) is a birnavirus that causes Gumboro, an acute and highly contagious disease that destroys B lymphocytes within the bursa of Fabricius, causing morbidity, mortality and immunosuppression, leaving birds susceptible to other infections and compromising vaccination programmes [13]. The IBDV major capsid protein VP2 is the target of antiviral neutralising antibodies [14-16] and is already used in a licenced HVT-vectored vaccine (Vaxxitec, Merial). Infectious laryngotracheitis virus (ILTV) is an alphaherpesvirus that causes highly contagious acute respiratory disease leading to growth depression, reduced egg production and death [17]. A fowlpox virus-vectored vaccine that expresses ILTV membrane glycoprotein B $(\mathrm{gB})$ and DNA packaging protein UL-32 is available (Ceva Bioimmune Vectormune ${ }^{\circ}$ FP-LT). Additionally, the ILTV membrane glycoproteins $\mathrm{gD}$ and gI have been expressed in the HVT-vectored vaccine Innovax-ILT (MSD Animal Heath).

Therefore, VP2 from a very virulent strain of IBDV (termed vvVP2) and gI from ILTV were each expressed in populations of transgenic E. tenella parasites under the control of E. tenella promoter sequences $5{ }^{\prime} E t$-Actin [9] or 5'Et-TIF (a genomic region upstream of gene ETH_00025365 encoding a putative translation initiation factor, E. Pegg, unpublished) in order to test the suitability of the parasite as an oral vaccine vehicle. The different transgenic populations were used to immunise chickens and their ability to develop any type of immune response was analysed.

\section{Methods}

\section{Parasites and birds}

The E. tenella Wisconsin strain was used throughout [18]. Oocyst purification, excystation and sporozoite purification were performed as described previously $[19,20]$. Parasites were propagated in 3 week-old White Leghorn chickens reared under specific pathogen free conditions and dosed with $4 \times 10^{3}$ sporulated oocysts (by oral intubation), or with $0.75 \times 10^{6}$ transfected sporozoites (by cloacal drinking [9]). Transfected sporozoites were also used to infect monolayers of Madin-Darby bovine kidney (MDBK; ECACC-Sigma-Aldrich, Salisbury, UK) cells as previously described [21].

Polymerase Chain Reaction (PCR) and molecular cloning PCR amplification was performed using Taq DNA Polymerase (Invitrogen, California, USA). Primers were designed using CLC Main Workbench software (CLC bio, Aarhus, Denmark) and synthesised by Sigma. Each PCR reaction contained DNA template, forward and reverse primers $(20 \mathrm{pmol}), \mathrm{Taq}$ polymerase $(0.5 \mathrm{U})$, dNTPs (0.2 mM; Promega, Hampshire, UK), Tris- $\mathrm{HCl}(20 \mathrm{mM})$, $\mathrm{KCl}(50 \mathrm{mM})$ and $\mathrm{MgCl}_{2}(2 \mathrm{mM})$. Standard cycle parameters were $1 \times\left(5 \mathrm{~min}\right.$ at $\left.95{ }^{\circ} \mathrm{C}\right), 30 \times\left(30 \mathrm{~s}\right.$ at $94{ }^{\circ} \mathrm{C}, 30 \mathrm{~s}$ at $56{ }^{\circ} \mathrm{C}$ and $1 \mathrm{~min}$ at $\left.72{ }^{\circ} \mathrm{C}\right)$ and $1 \times\left(10 \mathrm{~min}\right.$ at $\left.72{ }^{\circ} \mathrm{C}\right)$. Post-amplification PCR products were resolved by agarose gel electrophoresis, excised and purified using MinElute Gel Extraction (Qiagen, West Sussex, UK) and cloned into pGEM $^{\circ}-\mathrm{T}$ Easy (Promega). Plasmids were propagated in 
Escherichia coli XL1-Blue (Stratagene, California, USA), purified using QIAprep Spin Miniprep (Qiagen) and sequenced (GATC Biotech, Konstanz, Germany) as described by the respective manufacturers. Sequence analysis was done using CLC Main Workbench.

\section{Plasmid constructs for transgene expression in E. tenella} Starting plasmid pCIT_CjaA [10] contained the mCitrine coding sequence directly downstream of the $5^{\prime} E t-M i c 1$ promoter, and a second expression cassette where the viral genes of interest could be cloned downstream of either the $5^{\prime} E t$-Actin or $5^{\prime} E t$-TIF promoter region (Fig. 1).

To generate plasmids using the 5'Et-Actin promoter (Table 1, Fig. 1a), primers incorporating Xho I (for $v v V P 2$ and $g I$ ) or Xba I (for $m$ Cherry) and Bgl II restriction sites (Additional file 1: Table S1) were used to amplify the coding sequences of IBDV $v v V P 2$ (accession number HG974565.1; derived from F52/70 Faragher challenge virus, GDV-232/270890) and ILTV gI (accession number KP677883.1; derived from Innovax-ILT vaccine, HVT-138), combined in the plasmid pVEC211 (kindly provided by MSD Animal Health) and mCherry fluorescent reporter (from Core Construct 1; [9]). The PCR products were cloned into $\mathrm{pGEM}^{\circ} \mathrm{T}$ Easy (Promega) and digested with Xho I or Xba I and Bgl II (NEB, Hertfordshire, UK) for subsequent ligation into the pCIT_CjaA backbone, digested in parallel using the same endonucleases. For the second set of plasmids (Table 1, Fig. 1b), the $5^{\prime}$ Et-Actin promoter sequence was replaced by the ETH_00025365 (putative translation initiation factor TIF) promoter region, 5'Et-TIF. A region of $\sim 1 \mathrm{~kb}$ upstream of the ETH_00025365 coding sequence was amplified using primers including Cla I (for $\nu \nu V P 2$ ) or $B a m$ HI (for $g I$ and $m C h e r r y$ ) and $X b a$ I restriction sites (Additional file 1: Table S1). The PCR products were cloned into pGEM ${ }^{\circ}$-T Easy (Promega) and digested by Cla I or Bam HI and Xba I (NEB) for subsequent ligation into the corresponding plasmid. The correct sequence and insertions for each amplified product were confirmed by sequencing (GATC Biotech). Final plasmid DNAs were amplified in E. coli XL1-Blue (Stratagene), purified using an EndoFree Plasmid Maxi kit (Qiagen), linearized by digestion with Psi I (0.5 U per $1 \mu \mathrm{g}$ of plasmid DNA, NEB, confirmed by running out in an $0.8 \%$ agarose gel), precipitated in ethanolsodium acetate and quantified by NanoDrop (Thermo, Massachusetts, USA) as recommended by the respective manufacturers.

\section{Transfection of E. tenella sporozoites}

Transfection of purified sporozoites was carried out in 16-well strips using programme EO114 of a Nucleofector 4D (Lonza, Basel, Switzerland). Restriction enzymemediated integration (REMI) was applied to increase transfection efficiency $[9,22]$. For transfection, four wells were used, each containing $1 \times 10^{6}$ sporozoites in $20 \mu \mathrm{l}$ P3 buffer (Lonza), $12 \mu \mathrm{g}$ of plasmid DNA (in $2 \mu \mathrm{l}$ ) and 6 $\mathrm{U}$ Psi I. Each well was then shocked and $80 \mu \mathrm{l}$ of RPMI medium (Sigma, Suffolk, UK) was added per well and incubated at room temperature for $20 \mathrm{~min}$. Transfected sporozoites from the four wells were combined and used to infect MDBK cell cultures and/or to infect chickens.

Sporozoite survival rates were estimated after transfection using Trypan blue dye exclusion (Invitrogen). One million of the initial transfected sporozoites were incubated with MDBK cells at $41{ }^{\circ} \mathrm{C}-5 \% \mathrm{CO}_{2}$ in 24 well plates, washed with Advanced DMEM (Gibco, Leicestershire, UK) (supplemented with $2 \%$ of foetal bovine serum (FBS; Sigma) and $100 \mathrm{U}$ of Penicillin/Streptomycin (Fisher, Leicestershire, UK)) after $2 \mathrm{~h}$, and after $24 \mathrm{~h}$ the presence of parasites expressing mCitrine and/or mCherry was confirmed by fluorescent microscopy (Leica DMI3000B DCF365FX, Wetzlar, Germany).

\section{Obtaining populations of transgenic oocysts}

Chickens were infected with transfected sporozoites equivalent to $0.75 \times 10^{6}$ of the starting sporozoite sample by direct cloacal inoculation [9]. The precise live sporozoite dose was calculated subsequently based upon survival during transfection. After 1 week, oocysts were harvested from caecal contents and sporulated by incubation and agitation in potassium dichromate $(2 \% \mathrm{w} / \mathrm{v})$ for 3 days at $27{ }^{\circ} \mathrm{C}$. The proportion of fluorescent parasites in each oocyst population was determined by microscope counts (Leica DMI3000B - DCF365FX) and fluorescence-activated cell sorting (FACS) on a BD FACS Aria $^{\mathrm{TM}}$ III (BD, California, USA). Further passages of fluorescent-sorted sporulated oocysts through chickens were carried out to increase the proportion of transgenic parasites within the population.

Flow-activated cell sorting (FACS) of transgenic populations Expression of $m$ Citrine was used to identify parasites that were successfully transfected with test plasmids. Fluorescent oocysts were sorted in the BD FACS Aria ${ }^{\mathrm{m}}$ III (BD) using an excitation wavelength of $488 \mathrm{~nm}$, emission filter of 330/30, a 100 micron nozzle and pressure of 20 psi. Sample purity (fluorescent events/total events) was recorded for each population before and after sorting.

\section{Nucleic acid and protein isolation and complementary DNA synthesis}

TRIzol $^{\bullet}$ Regent (Invitrogen) was used for simultaneous isolation of RNA, genomic DNA (gDNA) and protein from oocysts. Two million sporulated oocysts were used and extractions were performed following the manufacturer's recommendations. RNA and protein pellets were 

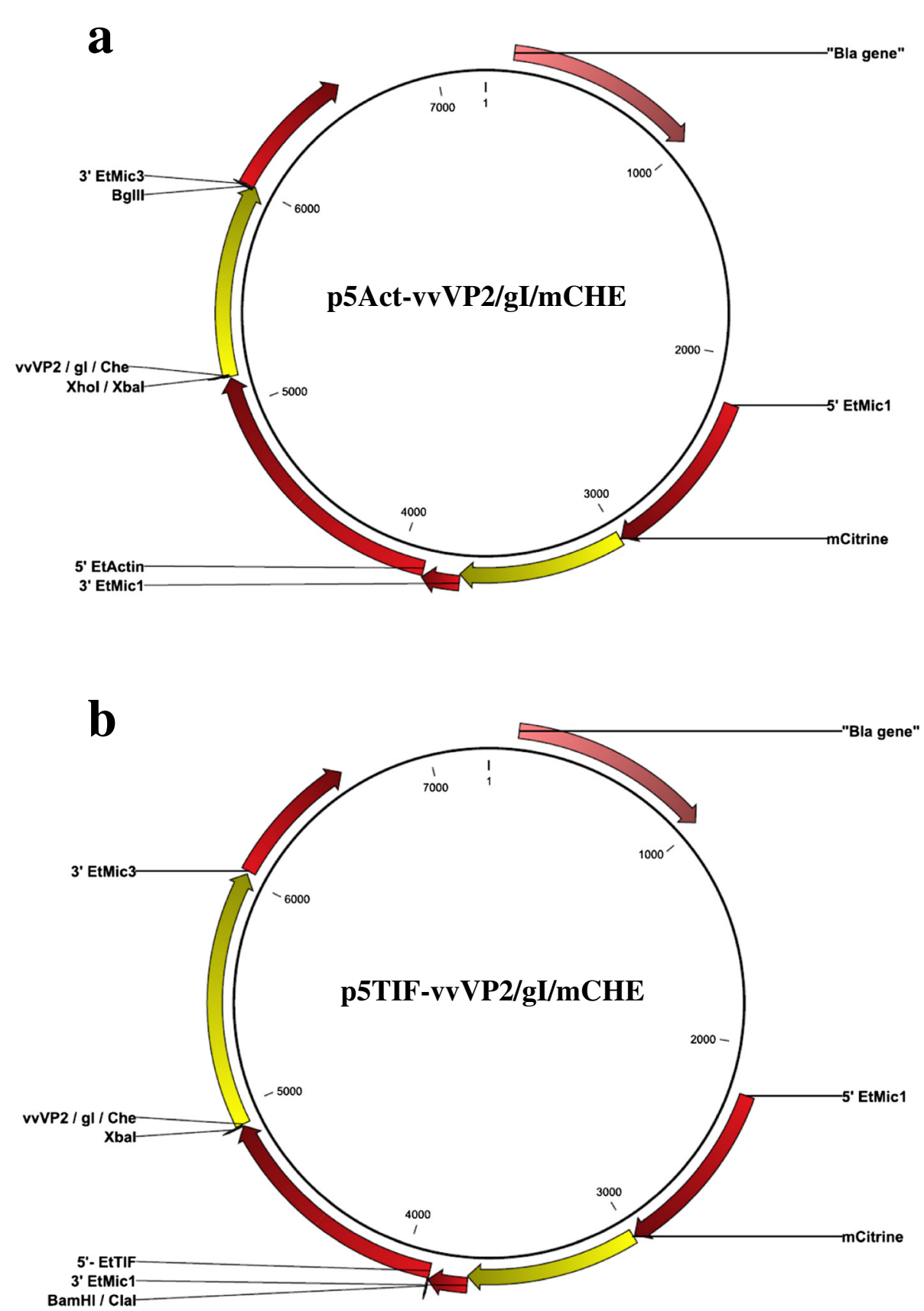

Fig. 1 Transfection constructs used for the genetic complementation of E. tenella with the viral genes VWVP2 (IBDV), gl (ILTV) or the reporter gene $m$ Cherry under the control of $\mathbf{a}$ the $5^{\prime} E t$-Actin promoter region or $\mathbf{b}$ the $5^{\prime} E t-T I F$ promoter region. The positions of restriction sites used for cloning the gene or promoter region are indicated for each plasmid

stored at $-80{ }^{\circ} \mathrm{C}$, gDNA at $-20{ }^{\circ} \mathrm{C}$, until further use. Complementary DNA (cDNA) was synthesized from total RNA using SuperScript $\mathrm{II}^{\circ}$ reverse transcriptase (200 U; Invitrogen), random hexamer primers $(2.5 \mu \mathrm{M}$; Applied Biosystems, Leicestershire, UK) and RNaseOUT ${ }^{\mathrm{Tm}}$ recombinant ribonuclease inhibitor (40U; Invitrogen) according to the manufacturer's instructions. cDNA was stored at $-20{ }^{\circ} \mathrm{C}$ until further use. To confirm the absence of
gDNA in the cDNA samples, primers for the Et-Actin gene were designed across an intron (Additional file 1: Table S1) such that gDNA contamination of cDNA would result in amplification of two fragments. The presence of transgene and transgene transcription was analysed for each oocyst population by PCR of gDNA and cDNA samples with specific primers (Additional file 1: Table S1). 
Table 1 Summary of plasmid constructs used for genetic complementation of E. tenella

\begin{tabular}{|c|c|c|c|}
\hline Plasmid & Fluorescent reporter & Target gene & Gene promoter \\
\hline p5Act-vvVP2 ${ }^{a}$ & 5'EtMic1-mCitrine-3'EtMic1 & VVVP2 (IBDV) & $5^{\prime} E t-A c t$ \\
\hline p5Act-gl ${ }^{a}$ & 5'EtMic1-mCitrine-3'EtMic1 & $g /($ ILTV) & $5^{\prime} E t-A c t$ \\
\hline p5Act-mChe ${ }^{b}$ & 5'EtMic1-mCitrine-3'EtMic1 & mCherry & $5^{\prime} E t-A c t$ \\
\hline p5TIF-VvVP2 ${ }^{a}$ & 5'EtMic1-mCitrine-3'EtMic1 & vVVP2 (IBDV) & $5^{\prime} E t-T I F$ \\
\hline p5TIF-gl $\left.\right|^{\mathrm{a}}$ & 5'EtMic1-mCitrine-3'EtMic1 & $g /($ ILTV) & $5^{\prime} E t-T I F$ \\
\hline p5TIF-mChe ${ }^{b}$ & 5'EtMic1-mCitrine-3'EtMic1 & mCherry & $5^{\prime} E t-T I F$ \\
\hline
\end{tabular}

${ }^{a}$ Used for the generation of stable populations

bUsed to test mCherry expression in transient transfection

\section{Real time quantitative PCR}

Real time quantitative PCR (qPCR) was performed in a CFX96 Touch ${ }^{\circledast}$ Real-Time PCR Detection System (Bio-Rad, Hertfordshire, UK) using DNA-binding dye SsoFastTM EvaGreen $^{\circledR}$ Supermix (Bio-Rad). The PCR mixture contained $1 \mu \mathrm{l}$ DNA template (gDNA or cDNA), SsoFast EvaGreen supermix $(10 \mu \mathrm{l})$, forward and reverse primers (250 nM) and RNase/DNase-free water in a final volume of $20 \mu \mathrm{l}$. Amplification was performed according to the manufacturer's protocol, with one cycle at $95{ }^{\circ} \mathrm{C}$ for $1 \mathrm{~min}$, followed by 40 cycles at $95{ }^{\circ} \mathrm{C}$ for $15 \mathrm{~s}$ and $60{ }^{\circ} \mathrm{C}$ for $30 \mathrm{~s}$. All samples were processed in triplicate, supplemented by a positive control dilution series and a no template negative control. Data were analysed with the Bio-Rad CFX Manager software (Bio-Rad).

The average copy number of transgenes per parasite genome was determined for each oocyst population using gDNA and specific primers for mCitrine (to estimate the transgene copy number) and Eimeria spp. $5 \mathrm{~S}$ rDNA (to estimate the total number of genomes) (Additional file 1: Table S1); as described previously [9].

Transgene transcription was quantified from cDNA using specific primers (Additional file 1: Table S1) and compared with serial dilutions of DNA standard templates for each transgene. pGEM $^{\bullet} \mathrm{T}$ Easy (Promega) plasmids containing the $v v V P 2, g I$ or mCitrine coding sequences were used as the DNA standard templates. Standard curves were prepared for all genes from $10^{8}$ to $10^{2}$ plasmid copies, against which transcript copy numbers were quantified. Data were analysed using the Kruskal-Wallis test followed by Mann-Whitney test for pairwise comparison (GraphPad Prism 6 v.6.00, California, USA).

\section{Expression of rec-vvVP2, rec-gl and rec-gD recombinant proteins}

Sequences corresponding to immunogenic regions (predicted by CLC Main Workbench software; CLC bio) of IBDV vvVP2 (nt199-nt657; accession number HG974565.1) and ILTV gI (nt420-nt810; KP677883.1) and ILTV gD (nt645-nt1182; KP677882.1) were amplified and cloned into plasmid vector pET32b (+) (Novagen, Hertfordshire, UK). DNA fragments were amplified from plasmid pVEC211 (kindly provided from MSD Animal Health) incorporating Hind III and Xho I restriction sites using the primers listed in Additional file 1: Table S1. PCR products were subcloned into $\mathrm{pGEM}^{-} \mathrm{-T}$ Easy (Promega) and digested by Hind III and Xho I (NEB) for ligation into pET32b(+), digested in parallel using the same endonucleases.

Recombinant proteins were expressed as polyhistidine (His6) and thioredoxin fusions in BL21 (DE3) pLysS E. coli (Stratagene). Bacteria were lysed overnight with phosphate buffer, $40 \mathrm{mM}$ imidazole, $5 \%$ (v/v) glycerol, $0.5 \%(\mathrm{v} / \mathrm{v})$ triton x 100, lysozyme (5KU/gr; Novagen) and benzonase $(25 \mathrm{U} / \mathrm{ml}$; Sigma). Insoluble recombinant proteins (rec-vvVP2 and rec-gI) were denatured in phosphate buffer containing $8 \mathrm{M}$ urea and $40 \mathrm{mM}$ imidazole. Recombinant proteins were purified by immobilized metal ion affinity chromatography (IMAC) using HisTrap ${ }^{\text {тм }} \mathrm{HP}$ columns following the manufacturers protocols (GE Healthcare, Buckinghamshire, UK). After elution in $500 \mathrm{mM}$ imidazole, recombinant proteins were checked by SDS-PAGE (Invitrogen) and protein concentration estimated by Bradford assay (Invitrogen) using serial dilutions of bovine serum albumin (BSA) as standard.

\section{Immunogenicity trial}

To evaluate the potential of E. tenella transgenic oocyst populations to stimulate specific antibody responses against an expressed transgene, groups of 21-day-old White Leghorn chickens were immunised orally with oocysts containing IBDV $v v V P 2$ or ILTV $g I$ transgenes under the control of either the 5'Et-Actin or $5^{\prime} E t-T I F$ promoter. Birds dosed with E. tenella wild type oocysts or with PBS were used as controls (Table 2). To mimic the re-cycling of parasites that occurs in natural infections, chickens were infected on more than one occasion, as stated in Table 2. Faeces were collected from each group of birds at 3 and 7 days after the first infection and oocysts were recovered to demonstrate successful inoculation of birds with transgenic or wild type E. tenella. Data were analysed by Chi-square test followed by Fisher's exact test (GraphPad Prism 6 v.6.00).

All birds were cardiac bled under terminal anaesthesia at the end of the study at 57 days of age (42 days after the last immunisation). Bloods were incubated at $37{ }^{\circ} \mathrm{C}$ for $2 \mathrm{~h}$, then transferred to $4{ }^{\circ} \mathrm{C}$ overnight and serum collected after centrifugation at $10,000 \mathrm{~g}$ for $10 \mathrm{~min}$ and stored at $-20{ }^{\circ} \mathrm{C}$ until further use.

\section{SDS-polyacrylamide gel electrophoresis (PAGE), Western blot and ELISA}

Total protein extracted by $\mathrm{TRIzol}^{\circledR}$ Reagent (Invitrogen) from passaged oocysts of transgenic or wild type parasites was electrophoresed through NuPAGE 4-12 \% 
Table 2 Experimental groups used for immunisation trial

\begin{tabular}{|c|c|c|c|c|c|c|}
\hline Group & Parasite population & No. of birds & 100 oocysts & 500 oocysts & 3000 oocysts & 5000 oocyst \\
\hline 1 & Et-Act-vvVP2-P6 & 12 & day 0 & day 4 & day 6 & day 13 \\
\hline 2 & Et-TIF-vvVP2-P2 & 12 & day 0 & day 4 & day 6 & day 13 \\
\hline 3 & E. tenella (wt) & 6 & day 0 & day 4 & day 6 & day 13 \\
\hline 4 & Et-Act-gl-P5 & 12 & day 0 & day 2 & day 8 & day 14 \\
\hline 5 & Et-TIF-gl-P2 & 12 & day 0 & day 2 & day 8 & day 14 \\
\hline 6 & E. tenella (wt) & 6 & day 0 & day 2 & day 8 & day 14 \\
\hline
\end{tabular}

Bis-Tris 10 well gels (Invitrogen) in Laemmli loading buffer (Sigma). Proteins were transferred to nitrocellulose membranes (GE Healthcare) in a semidry system following the manufacturers protocols (Invitrogen) and blocked in $5 \%(\mathrm{w} / \mathrm{v})$ blotting grade non-fat milk (BioRad) overnight. Membranes were incubated for 1 hour with a 1/100 dilution of a set of sera kindly provided by MSD Animal Health: anti-mouse MCA-LTV-Mab6INT and anti-chicken ILT Spafas for gI; anti-mouse MCA 10-INT and anti-mouse IBDV R63 MoAb for vvVP2. After washing three times with TBS-Tween $0.05 \%(\mathrm{v} / \mathrm{v})$ membranes were incubated for 1 hour with goat anti-mouse IgG antibody horseradish peroxidase (HRP) conjugate (Merk Millipore, Hertfordshire, UK) or rabbit anti-chicken IgG antibody HRP conjugate (Merk Millipore). Membranes were washed three times with TBS-Tween $0.05 \%(\mathrm{v} / \mathrm{v})$, once with TBS and finally with distilled water before adding Luminata substrate (Merck Millipore). Chemiluminiscence was visualised in a G:BOX (Syngene, Cambridge, UK) and images were taken with GeneSnap 7.12 software (Syngene).

To determine whether immunised chickens developed specific antibodies against vvVP2, gI or E. tenella, $5 \mu \mathrm{g}$ of rec-vvVP2 or rec-gI recombinant proteins or $10^{7} E$. tenella sporozoites were electrophoresed through NuPAGE 4-12 \% Bis-Tris 1 well gels (Invitrogen) in Laemmli loading buffer (Sigma). Proteins were transferred and blocked as described above. The membranes were incubated with different dilutions of chicken serum $(1 / 200-1 / 100-1 / 50)$ and corresponding controls (sera from chickens immunised with Vaxxitec for vvVP2 and with ILT Spafas for gI) in a multiscreen apparatus (BioRad) for 1 hour, washed three times with TBS-Tween $0.05 \%(\mathrm{v} / \mathrm{v})$ and incubated again for 1 hour with rabbit anti-chicken IgG antibody HRP conjugate (Merck Millipore). Membranes were washed and developed as described above. Antisera were also analysed by enzymelinked immunosorbent assay (ELISA) at The Service Laboratory (Boxmeer, Netherlands) using an IDEXX IBD Antibody Test Kit for vvVP2 and the ProFLOK ${ }^{\oplus}$ ITL Antibody Test Kit. The presence of neutralising antibodies for vvVP2 were also analysed using the GDV D78 $\mathrm{VN}$ assay.

\section{Lymphoproliferation assays}

An experiment replicating the immunization schedule of the immunogenicity assay (as above and Table 2) using 7-day-old chickens, was performed to analyse the ability of vvVP2 and gI antigens, expressed by E. tenella, to induce lymphocyte proliferation. In this experiment groups of four chickens were used. For peripheral blood mononuclear cells (PBMC), birds were bled from the wing vein at 7 and 14 days after the last immunization; blood was treated with heparin to avoid coagulation $(0.3 \mathrm{U} / \mu \mathrm{l})$ and $1 \mathrm{ml}$ was mixed with Histopaque 1077 (Sigma) (1:1). For splenocytes, spleens were removed at post-mortem (21 days after the last immunization), sliced, washed with PBS, passed through a cell strainer $(40 \mu \mathrm{m})$ and the homogenate $(6 \mathrm{ml})$ was mixed with Histopaque 1077 (Sigma) (1:1). Samples were centrifuged at $1250 \mathrm{~g}$ for $30 \mathrm{~min}$ and the interphase recovered and washed with $1 \mathrm{ml}$ (PBMC) or $5 \mathrm{ml}$ (splenocytes) of PBS three times (600 g - $15 \mathrm{~min})$, then resuspended in $1 \mathrm{ml}$ or $5 \mathrm{ml}$ RPMI containing $10 \%$ $(\mathrm{v} / \mathrm{v})$ FBS. Isolated cells were counted and $10^{5}$ cells added per well in 96-well-plates, using 12 wells per group.

PBMC and splenocytes were stimulated with Concanavalin A (ConA; $5 \mu \mathrm{g} / \mathrm{ml}$; Sigma), soluble E. tenella antigen $(10 \mu \mathrm{g} / \mathrm{ml})$, recombinant protein (vvVP2 or gI; $10 \mu \mathrm{g} / \mathrm{ml}$ ) or PBS and incubated at $41{ }^{\circ} \mathrm{C}$ with $5 \% \mathrm{CO}_{2}$ for 5 days. Cell proliferation was monitored by the incorporation of tritiated thymidine $\left({ }^{3} \mathrm{H}-\mathrm{Tdr}\right.$, specific activity $485 \mathrm{GBq} / \mathrm{mmol}$; Thermo), $37 \mathrm{kBq}{ }^{3} \mathrm{H}$-Tdr were added for the final $24 \mathrm{~h}$ of incubation. Plates were then stored at $-20{ }^{\circ} \mathrm{C}$ for at least 1 day and cells were harvested onto with a semi-automated cell harvester (Skatron, California, USA) and counted in a 1205 Betaplate $^{{ }^{\text {тм }}}$ counter (Wallac, Zurich, Switzerland). Data were analysed by a one-way ANOVA with post-hoc Bonferroni test (SPSS Statistics 22, New York, USA).

\section{Results}

Transiently transfected $E$. tenella sporozoites expressed foreign antigens under the regulation of two different promoter regions

The functionality of promoters $5^{\prime} E t$-Actin and $5^{\prime} E t-T I F$ was confirmed by detection of red fluorescence in sporozoites transfected with constructs containing mCherry 
cloned downstream of each promoter (Table 1, Fig. 2a). Quantification of transgene copy number per parasite genome and the level of transgene transcription was not carried out in these short-term sporozoite cultures because non-integrated plasmid was also detected, evidenced by a number of mCitrine copies per genome determined by qPCR to exceed 35,000 (data not shown).

\section{Viral proteins were expressed in transgenic populations} of sporulated oocysts, and transgene copy number per parasite genome reduced after in vivo parasite passage Sporozoites transfected with the different plasmids (Table 1) showed a survival between 17.5 and $24.0 \%$ and were used to infect chickens and generate oocysts (Table 3). The populations that emerged contained mixtures of fluorescent and non-fluorescent oocysts. Each population was enriched for fluorescent oocysts after sporulation by FACS sorting (85-95\% purity) and repassaged in chickens on two to six occasions per plasmid (Table 3, Fig. 2b). The proportion of fluorescent oocysts increased after passaging, but did not exceed $22 \%$ in any example (Fig. 3a).

The presence and expression of mCitrine in transgenic parasites was evidenced by fluorescence (Fig. 2b). The presence of the second cassette containing each foreign gene was confirmed by PCR with gene-specific primers (Additional file 1: Table S1). These same primers were also used to confirm transgene transcription by real time PCR (RT-PCR).

Variation in the number of transgene copies per genome was analysed for each generation of transgenic parasites. The transgene copy number dropped dramatically after two passages, and thereafter further passages were done until they stabilised at an average of less than ten copies per genome (Fig. 3b).

To determine the occurrence of transgene expression a band of the expected size $(\sim 62 \mathrm{kDa})$ was detected by Western blotting using extracts of transgenic populations expressing gI (Et-Act-gI and Et-TIF-gI) when they were incubated with MCA-LTV-Mab6-INT (Fig. 3c). A second band of $\sim 56 \mathrm{KDa}$ was also detected in both transgenic populations but was absent from the wildtype parasites. Recognition with a second antibody, ILT Spafas, was not conclusive (Fig. 3c). In contrast no clear recognition of a protein of the expected size $(\sim 42 \mathrm{kDa})$ was observed in extracts of transgenic populations expressing vvVP2 (Et-Act-vvVP2 and Et-TIF-vvVP2) (Fig. 3c), only an unspecific band $(\sim 59 \mathrm{kDa})$ which was conserved within the wild-type sample was observed.

\section{Transcription levels of viral transgenes in sporulated} oocysts were independent of the promoter region used Each plasmid carried two coding sequences under the regulation of different promoters, 5'Et-Mic1 linked to $m$ Citrine and $5^{\prime} E t$-Actin or $5^{\prime} E t$-TIF linked to $\nu v V P 2$ or $g I$. In order to determine the relative level of transcription of $5^{\prime} E t-A c t i n$ or $5^{\prime} E t-T I F$ in relation to $5^{\prime} E t-M i c 1$ a reverse transcription $\mathrm{qPCR}$ was performed. The transcription of $m$ Citrine linked to 5'Et-Mic1 was always higher than transcription of $\nu v V P 2$ or $g I$ linked to either 5'Et-Actin or 5'Et'TIF (Table 4). When expressing $g I$, $5^{\prime} E t^{\prime} T I F$ lead to a stronger transcription than $5^{\prime}$ Et-Actin

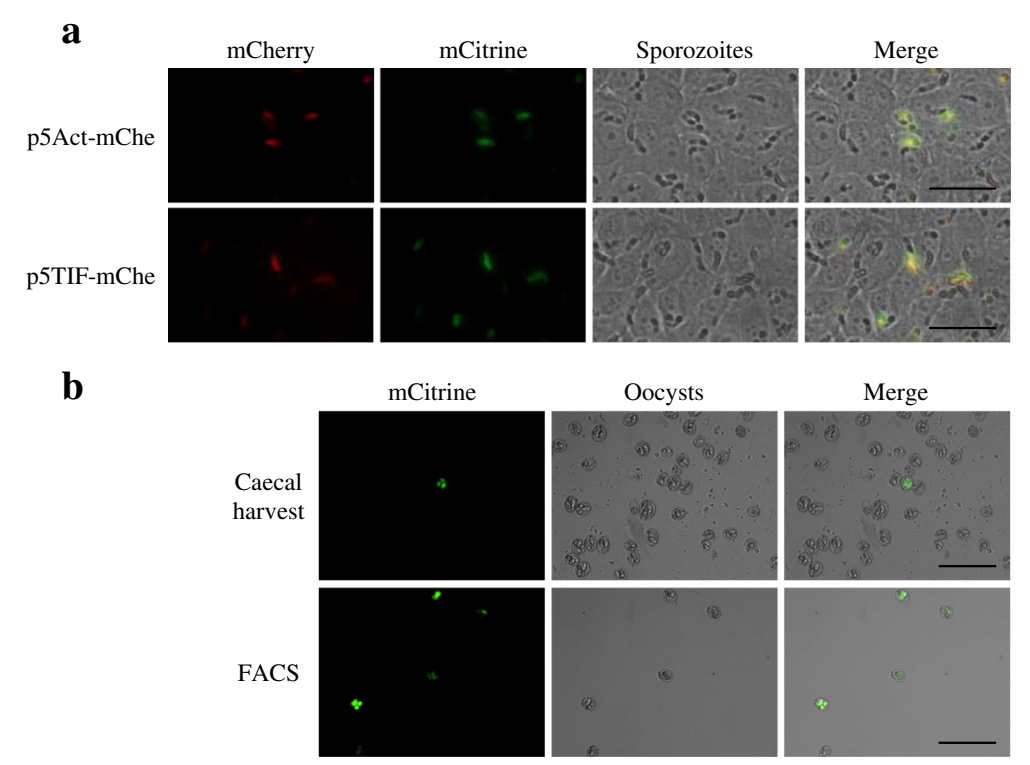

Fig. 2 Transgenic parasites expressing fluorescent reporters. a Transiently transfected sporozoites expressing mCherry under the control of 5'Et-Actin (panel above) or 5'Et-TIF (panel below) and mCitrine under the control of 5'Et-Mic1. Scale-bars: 40 um. b Oocysts recovered by caecal harvest following in vivo passage (panel above) and after enrichment by FACS (panel below). Scale-bars: $100 \mu \mathrm{m}$ 
Table 3 Sporozoite survival after transfection, the numbers used to initiate the primary infection and the total number of generations produced for each plasmid

\begin{tabular}{llll}
\hline Transfected plasmid & Parasite survival (\%) & Number of live sporozoites dosed per chicken & Number of in vivo passages \\
\hline p5Act-vVVP2 & 17.5 & 131,250 & 6 \\
p5Act-gl & 24.0 & 180,000 & 5 \\
p5TIF-VVVP2 & 21.6 & 162,080 & 2 \\
p5TIF-gl & 22.4 & 168,341 & 2 \\
\hline
\end{tabular}

(an average of 2.1 times more). However, when expressing $v v V P 2$, the promoter region 5 'Et-Actin led to a stronger transcription than $5^{\prime} E t^{\prime} T I F$ (an average 9.4 times more).

Immunisation of chickens with transgenic parasites led to the generation of specific antibodies but did not trigger lymphocyte proliferation in a re-stimulation assay

The presence of specific antibodies 42 days after the last immunisation against rec-vvVP2 or rec-gI was tested by Western blot for all groups of chickens immunised using the relevant transgenic parasite population, with between one and eight of twelve birds found to be positive per group (Fig. 4a). However, the sera were reactive only at high concentrations (Fig. 4b), none were positive when tested with commercial ELISA kits and the ELISA specific for virus neutralising antibodies resulted in a $2 \log$ titre of $<5$ (equivalent to a titre of less than 1 in 32; Additional file 1: Table S2). The frequency of antibody detection by Western blot was significantly higher for the group immunised with Et-TIF-gI compared to that immunised with Et-TIF-vvVP2 $(P<0.05$, Fisher's exact test), but there were no other significant differences found between any other groups. All sera from immunised chickens, whether positive or negative against the recombinant proteins, were positive when tested against $E$. tenella extracts (Additional file 1: Figure S1).

PBMC from birds immunised with transgenic parasites expressing vvVP2 (Et-Act-vvVP2 and Et-TIF-vvVP2) were isolated and re-stimulated in vitro at day 7 and 14 post-immunisation. Proliferation was observed when they were stimulated with the unspecific stimulator ConA (data not shown) or with E. tenella soluble antigen only at day 7. However, there were no significant changes $(P>0.05$, one-way ANOVA) when rec-vvVP2 was added to the media compared with the PBS controls or a non-specific recombinant protein (rec-gD) (Fig. 5). Birds immunised with transgenic parasites expressing gI (Et-Act-gI and Et-TIF-gI) were not tested at these time points. Splenocytes from birds of all immunised groups were isolated and stimulated at day 21 post-immunisation. Similar to what was observed for the PBMC, only ConA (data not shown) and E. tenella soluble antigen were able to stimulate the cells (Fig. 5).

\section{Discussion}

In this study new transgenic populations of E. tenella expressing antigens from either of two viruses that cause serious diseases in chickens, IBDV and ILTV, have been generated. Oral immunisation of chickens using these parasites was able to induce specific antibody responses against the viral antigens in some, but not all individuals. The idea of using Eimeria parasites as multivalent vaccine vectors was suggested several years ago, and to date some partial success has been reported. Clark et al. [10] expressed Campylobacter jejuni antigen CjaA in E. tenella and whilst birds immunised with the transgenic parasites were protected ( $\sim 90 \%$ reduction in C. jejuni in the caeca 2 weeks after challenge, compared to controls) evidence of immunological recognition of the antigen by the chicken was inconclusive. Moreover, Liu et al. [11] generated parasites expressing the M2 protein of the avian influenza virus, but found no evidence of antibody or cellular responses. The only immune responses reported in chickens raised by transgenic Eimeria has been against a fluorescent reporter protein (EYFP) [23], therefore, this is the first time that antibodies against viral proteins expressed in transgenic Eimeria have been reported. The antibodies were detected by Western blotting only when sera were used at low dilution and none were positive in standard commercial ELISA kits, which use sera at higher dilutions. Nevertheless, these results are significant since they demonstrate that the viral antigens were expressed and available to the chicken immune system. Improving the consistency of immunisation remains a major challenge.

The integration and transcription of transgenes, both for vvVP2 or gI, was shown in all the transgenic populations and for gI it was possible by Western blotting to identify proteins of the expected mass within extracts of sporulated oocysts. Antibodies against gI recognised two bands in the parasite extract and these could potentially correspond to unmodified and modified forms. Unmodified and $\mathrm{N}$-glycosylated forms have been described for native viral gI [24]. The E. tenella proteome has been shown to include multiple proteins associated with glycosylation, including $\mathrm{N}$-glycosylation [25], and while the modifications may not be identical in the two organisms, post-translational changes may explain the occurrence of two bands in transgenic, but not wild-type parasites. For 

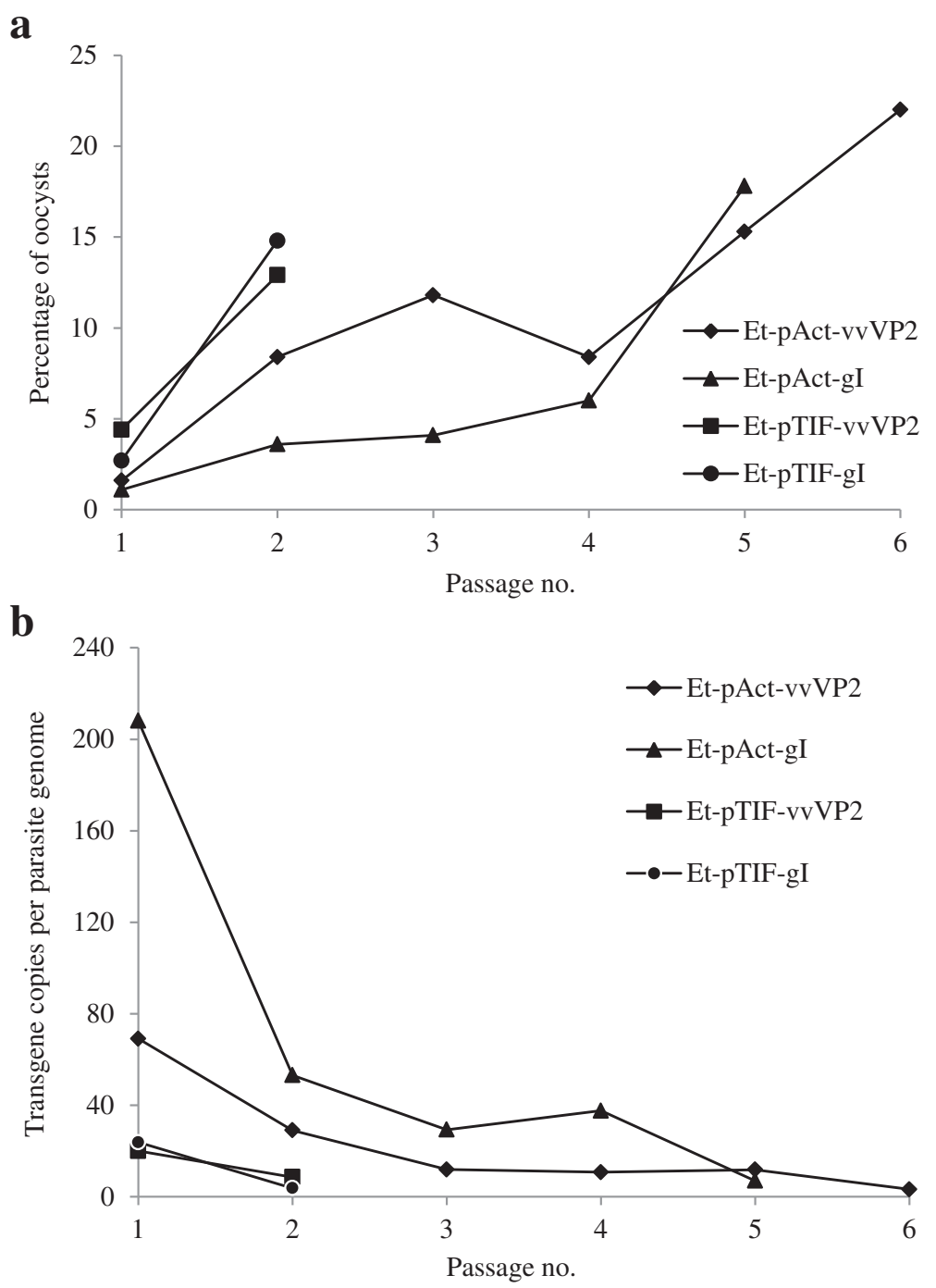

c

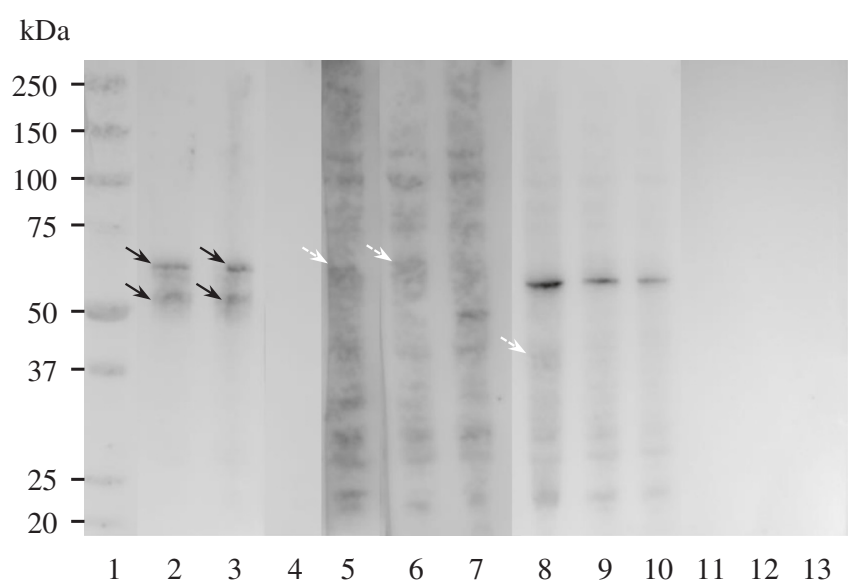

Fig. 3 (See legend on next page.) 
(See figure on previous page.)

Fig. 3 Analysis of transgenic E. tenella populations. a The percentage fluorescence detected for each transgenic population with successive in vivo passage. $\mathbf{b}$ Number of transgene copies per parasite genome for each transgenic population with successive passage through chickens. The number of copies was calculated using a standard curve of E. tenella gDNA. c Western blot of the different transgenic populations; Et-Act-gl-P5: lanes 2, 5; Et-TIF-gI-P2: lanes 3, 6; Et-Act-WVPP2-P6: lanes 8, 11; Et-TIF-VVVP2-P2: lanes 9, 12; and E. tenella (wt): lanes 4, 7, 10, 13; incubated with MCA-LTVMab6-INT (lanes 2-4) or ILT Spafas (lanes 5-7) for gl recognition and MCA 10-INT (lanes 8-10) or IBDV R63 MoAb (lanes 11-13) for vvVP2 recognition. Lane 1 is the Mw. Solid black arrows indicate specific band recognition with a size of $\sim 62 \mathrm{kDa}$ for gl and a processed form of it $\sim 56 \mathrm{kDa}$ (lines 2 and 3). Dotted white arrows indicate potential band recognition with sizes of $\sim 62 \mathrm{kDa}$ for gl and $\sim 42 \mathrm{kDa}$ for $\mathrm{WVP2}$ (lines 5, 6 and 8)

vvVP2, we could not directly prove protein expression in the parasites by Western blot because the antibodies expected to react with vvVP2 failed to recognise any form of recombinant vvVP2 cloned and produced in this same study. We infer vvVP2 expression by indirect methods including visualisation of mCherry expressed in the same vector frame, detection of vvVP2 transcripts and the presence of specific antibodies in immunised chickens.

The high sera concentration necessary for detection of specific antibodies against vvVP2 or gI, may relate to the total amount of foreign antigen being exposed to the chickens immune system. The immunisations in this study were done following protocols for vaccinations against Eimeria, in which the maximum number of oocysts inoculated was 5000 in the last immunisation. These numbers are considerably lower than those used with other live vectors such as Salmonella Typhimurium, for which doses from $10^{7}$ to $10^{9}$ organisms expressing VP2 were dosed [26]. Therefore, an interesting option could be the transference of these transgenic technologies to more fecund Eimeria species such as E. acervulina or E. mitis [27] in order to increase the number of transgene expressing organisms per immunisation.

Further, the transgenic populations used for the immunisations were unstable with respect to transgene retention and expression. Chickens were infected with populations enriched by FACS to contain between $85 \%$

Table 4 Relative transcription levels of the foreign gene in cassette 2 ( $V V V P 2$ or gl) compared with mCitrine in cassette 1 in different transgenic populations

\begin{tabular}{lcll}
\hline Population & mCitrine/vVVP2 $^{c}$ & Population & mCitrine/gl $^{\mathrm{c}}$ \\
\hline Et-Act-vVVP2-P1 & $34.7^{\mathrm{a}}$ & Et-Act-gl-P1 & $139.0^{\mathrm{a}}$ \\
Et-Act-vVVP2-P2 & $4.2^{\mathrm{a}}$ & Et-Act-gl-P2 & $36.3^{\mathrm{a}}$ \\
Et-Act-vVVP2-P3 & $4.2^{\mathrm{a}}$ & Et-Act-gl-P3 & $82.6^{\mathrm{a}}$ \\
Et-Act-vVVP2-P4 & $6.4^{\mathrm{a}}$ & Et-Act-gl-P4 & $62.8^{\mathrm{a}}$ \\
Et-Act-vVVP2-P5 & $1.7^{\mathrm{a}}$ & Et-Act-gl-P5 & $182.8^{\mathrm{a}}$ \\
Et-Act-vVVP2-P6 & $0.4^{\mathrm{a}}$ & & \\
Et-TIF-VVVP2-P1 & $67.2^{\mathrm{b}}$ & Et-TIF-gl-P1 & $76.9^{\mathrm{b}}$ \\
Et-TIF-VVVP2-P2 & $94.9^{\mathrm{b}}$ & Et-TIF-gl-P2 & $17.4^{\mathrm{b}}$ \\
\hline
\end{tabular}

${ }^{a}$ Equivalent to $5^{\prime} E t-M i c 1 / 5^{\prime} E t-A c t i n$ ratio

${ }^{b}$ Equivalent to $5^{\prime}$ Et-Mic1 / 5'Et-TIF ratio

${ }^{\mathrm{C}}$ Ratio indicates the times $m$ Citrine is higher than the other transcript and $95 \%$ fluorescent oocysts, however the harvested oocyst output collected after each passage always contained less than $22 \%$ fluorescent oocysts. Some possible reasons for this instability have been discussed previously by Clark et al. [9], including genetic segregation and recombination leading to transgene loss. Working with stable lines of transgenic parasites which maintain transgene expression after every passage might improve the efficacy of vaccination, although the difficulties associated with obtaining clonal lines precludes routine use during medium throughput testing. Specifically, Eimeria cannot be cloned in vitro by serial dilution or similar procedures. Instead, single sporocyst infection of chickens is needed to clone Eimeria, which is a time-consuming and inefficient process [11, 28]. Non-clonal populations that have $100 \%$ transgenic parasites can be obtained by applying drug selection over several in vivo passages to parasites where a drug-resistance cassette has been incorporated in the transfection process [9]; however, inclusion of drug-resistant genes is not appropriate for populations to be administrated as live vaccines in field chicken populations.

The choice of promoters to control timing and level of expression of the downstream transgene could be important for optimising recognition of foreign antigens by the host immune system. We characterised parasite populations expressing $v v V P 2$ or gI using $5^{\prime}$ Et-Actin, a constitutive promoter used previously $[9,28]$ or $5^{\prime} E t-T I F$, a novel promoter identified from an RNAseq database [29] that is linked to a gene transcribed $\sim 10$ fold higher than Et-Actin. However when transgene transcripts ( $\nu v V P 2$ or $g I)$ under the control of the two different promoters ( $5^{\prime} E t$-Actin or $\left.5^{\prime} E t-T I F\right)$ were quantified, in relation to mCitrine expressed under control of $5^{\prime} E t-M i c 1$ on the same plasmid, $5^{\prime} E t-T I F$ was found to promote higher transcription for $g I$, but not for $\nu \nu V P 2$, suggesting that the downstream transgene could be affecting the transcription regulation.

Protection against viral infections such as IBDV and ILTV can be achieved by the induction of a high titre of neutralising antibodies as demonstrated using the VP2 antigen for IBDV [30, 31], in contrast to more complicated immune mechanisms necessary to provide protection against other pathogens (eg. Clostridium perfringens [32]). While we have shown that the Eimeria expression 


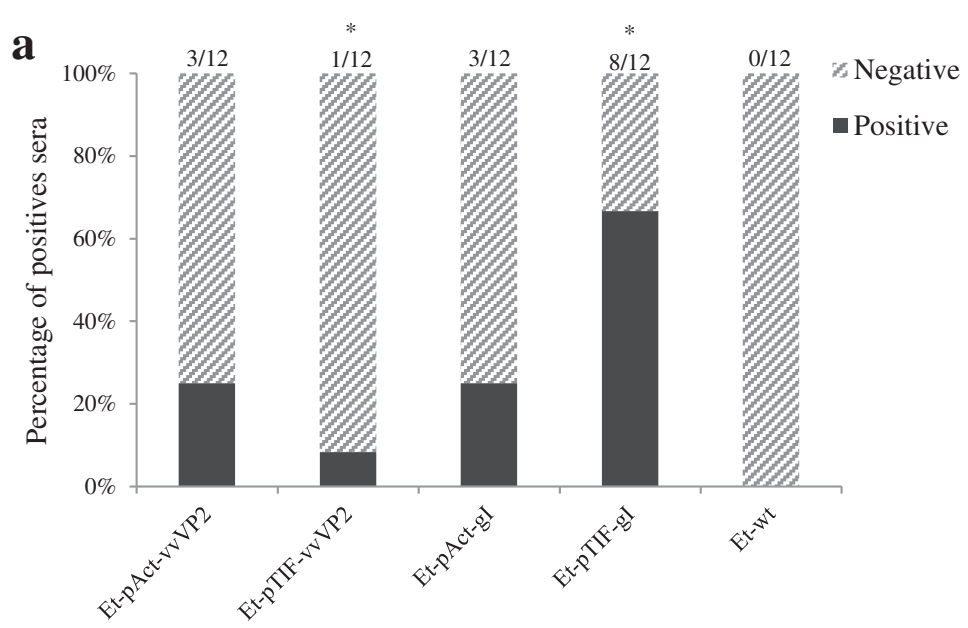

Transgenic populations

b
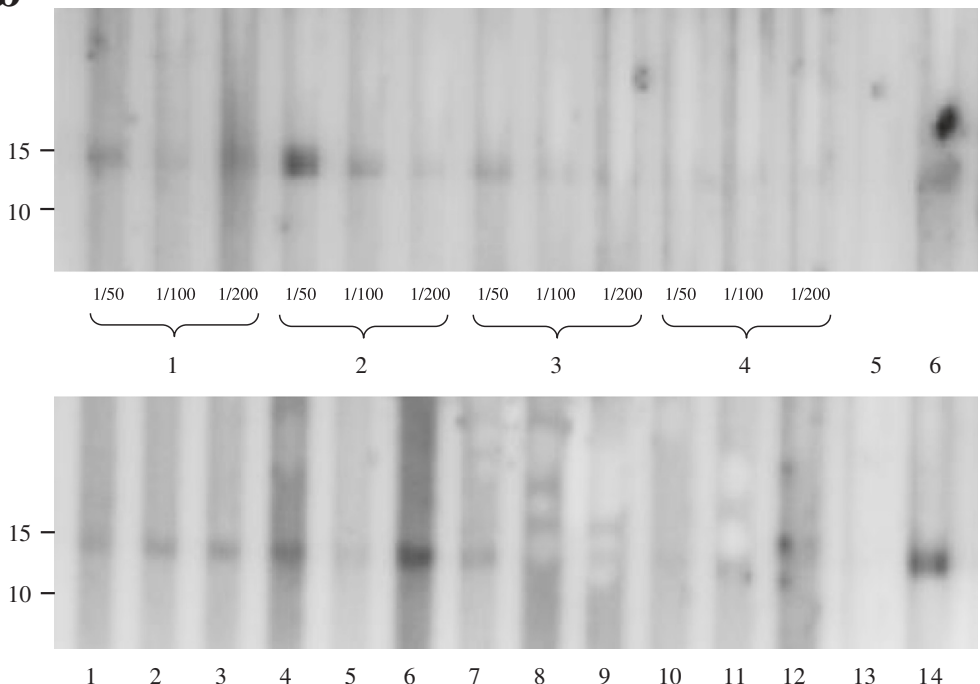

Fig. 4 Humoral immune responses by chickens immunised with E. tenella transgenic populations and wild type parasites. a Proportion of chickens presenting a positive reaction against rec-VVVP2 or rec-gl by western blot. ${ }^{*}$ Indicates significant differences between groups in the frequency of recombinant protein detection $(P<0.05$, Fisher's exact test). $\mathbf{b}$ Western blot of chicken sera against rec-vVVP2 (panel above); lanes 1-2: positive sera from chickens immunised with Et-Act-WVVP2-P6 at dilutions 1/50, 1/100 or 1/200; lanes 3-4: positive and negative sera from chickens immunised with Et-TIF-VVVP2-P2 at dilutions 1/50, 1/100 or 1/200; lane 5: negative control (sera from chickens immunised with E. tenella (wt) at dilution 1/50); lane 6: positive control (sera from chickens immunised with Vaxxitec at dilution 1/100) and Western blot of chicken sera against rec-gl (panel below); lanes 1-7: chicken reacting positive (1-3: Et-Act-gl-P5 at dilution 1/50; 4-7: Et-TIF-gl-P2 at dilution 1/50); lanes 8-12: chicken reacting negative (8-9: Et-Act-gl-P5 at dilution 1/50; 10-12: Et-TIF-gl-P2 at dilution 1/50); lane 13: negative control (sera from chickens immunised with E. tenella (wt) at dilution 1/50); lane 14: positive control (anti-chicken ILT Spafas at dilution 1/200). Rabbit anti-chicken IgG antibody HRP conjugate (Merk Millipore) at 1/1000 dilution was used as secondary antibody

system can be used to stimulate host immune recognition of foreign antigens, the levels of humoral response generated are likely to be insufficient to guarantee protection against challenge [33]. Prior to conducting an in vivo challenge the consistency and magnitude of immune response stimulated by transgenic Eimeria vaccination should be improved. Options include the use of promoters capable of inducing stronger transgene expression, or addition of targeting signals for delivery to the cell surface or secretion, which could to improve the exposition of the antigen and therefore its recognition [23, 34]. Optimization of the number of parasites per vaccinating dose or transference of the technology to more fecund Eimeria species might also encourage stimulation of more significant immune response. Novel technologies for directed integration such as CRISPR/Cas9 [35, 36] or the generation of clonal lines could also offer some advantages, improving accurate characterization of transgenic parasites and improving uniformity. 

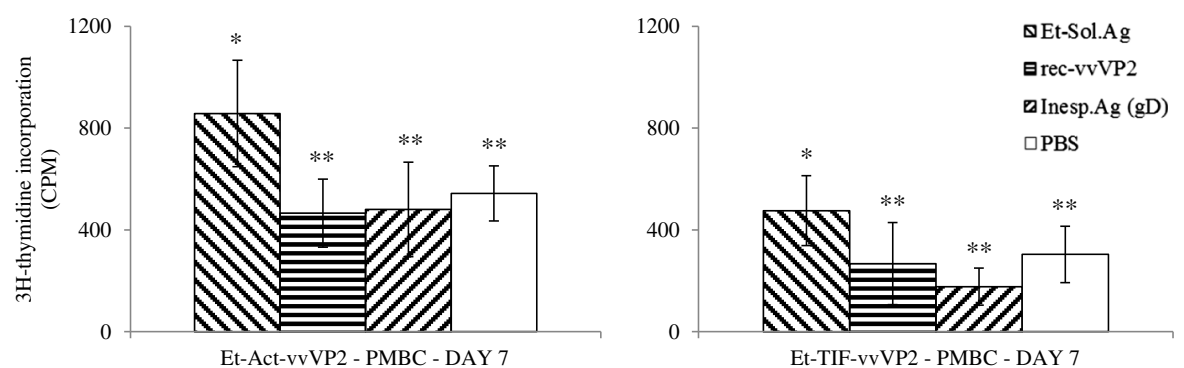

Et-TIF-vvVP2 - PMBC - DAY 7
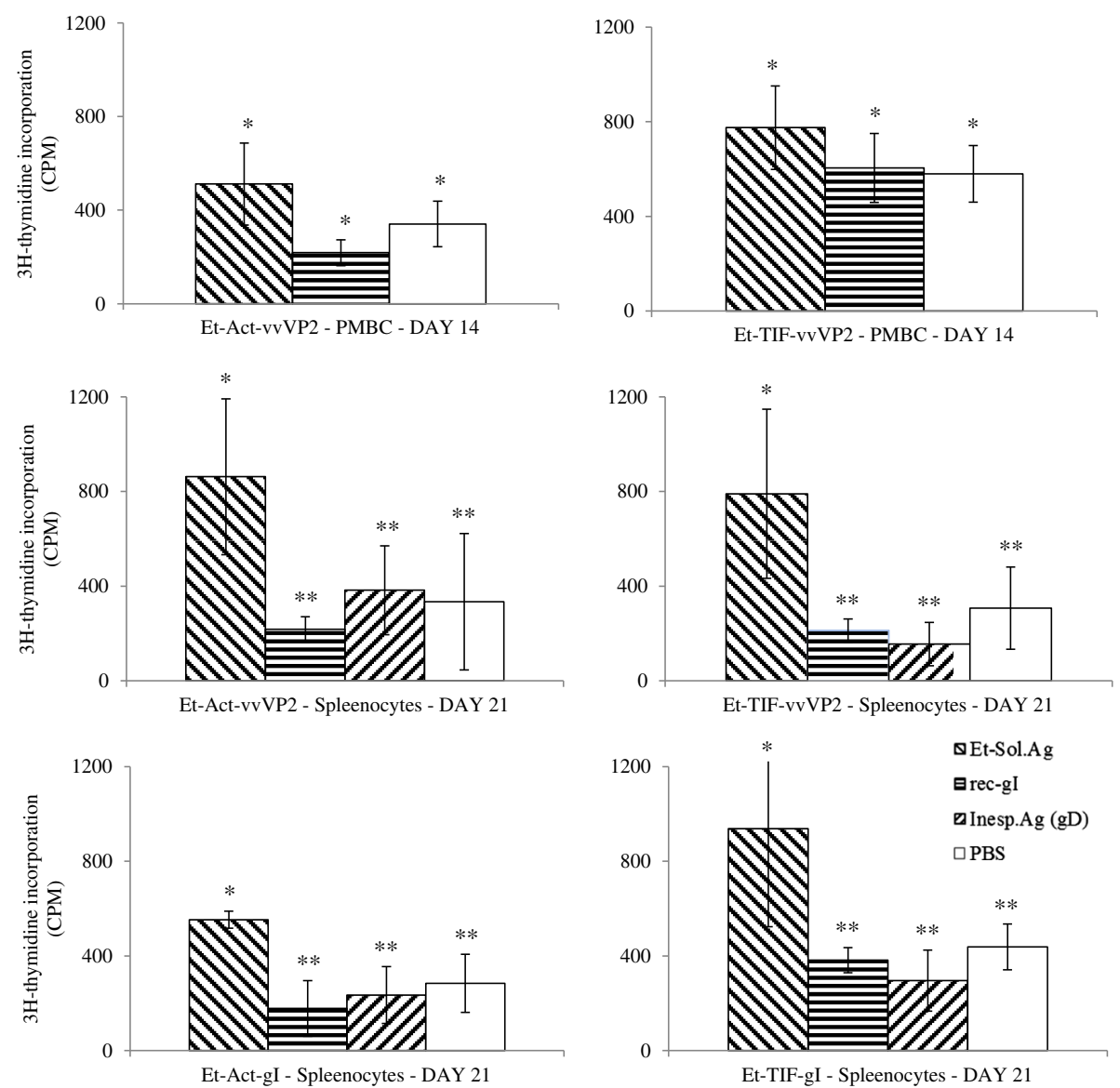

Fig. 5 Proliferation of PMBC and splenocytes isolated from chickens immunised with the different transgenic populations (Et-Act-VVVP2, Et-TIF-VVVP2, Et-Act-gl or Et-TIF-gl) after stimulation with E. tenella soluble antigens, the corresponding recombinant antigen (rec-vVVP2 or rec-gl), an unspecific antigen (rec-gD) or PBS. ${ }^{*},{ }^{* *}$ indicate different significances, only E. tenella soluble extract induced linfoproliferation at days 7 (Et-Act-vVVP2: $F_{(3,46)}=11.825, P<0.00001 ; E t-T I F-V V V P 2: F_{(3,45)}=9.041, P<0.00001$; one-way ANOVA) and 21 (Et-Act-VVVP2: $F_{(3,24)}=12.439, P<0.00001 ; E t-T I F-$ vvVP2: $F_{(3,42)}=9.470, P<0.00001 ; E t$-Act-gl: $F_{(3,35)}=11.769, P<0.00001 ; E t-T I F-g l: F_{(3,28)}=11.870, P<0.00001$; one-way ANOVA). No significant linfoproliferation was observed at day 14 (Et-Act-VVVP2: $F_{(2,10)}=2.557, P=0.127 ; E t-T I F-V V V P 2: F_{(2,27)}=1.091, P=0.350$; one-way ANOVA)

\section{Conclusions}

Newly generated transgenic populations of E. tenella are capable of expressing viral antigens vvVP2 from IBDV and gI from ILTV. Moreover, these expressed antigens were recognised by the chicken immune system as evidenced by the presence of specific antibodies in the sera, providing the first report of specific antigen recognition from viral avian pathogens expressed by transgenic Eimeria. Nonetheless, the low level response detected indicates a requirement of improve transgene expression before testing as a vaccine.

\section{Additional file}

Additional file 1: Table S1. Primers used in the study. Table S2. Analysis of post infection serum. Figure S1. Western blot of total E. tenella sporozoite extract against chicken sera immunised by infection with different transgenic or wild-type populations of E. tenella. Lane 1: Et-Act-wVVP2-P6; lane 
2: Et-TIF-WVP2-P2; lane 3: Et-Act-gl -P5; lane 4: Et-TIF-gl-P2; lane 5: E. tenella (wt): lanes 6-7: non-immunised; lane 8: positive control (anti-EtMic2); lane 9: Coomassie blue of E. tenella sporozoite extract; lane 10: Mw. Rabbit anti-chicken IgG antibody HRP conjugate or goat anti-mouse lgG antibody HRP (for EtMic2) conjugate at 1/1000 dilution were used as secondary antibody. (DOCX $3094 \mathrm{~kb})$

\section{Acknowledgments}

The authors wish to thank Amanda Gibson for the help with the FACS and Dirk Werling for advice and help with the lymphoproliferation assays. We would also like to thank Fran Benstead, Alison Riley and Philip Davies for technical assistance and advice. This manuscript has been assigned the reference PPB_01300 by the Royal Veterinary College.

\section{Funding}

This work was funded by an Industrial Partnership Award (IPA) from the Biotechnology and Biological Sciences Research Council (BBSRC) in partnership MSD Animal Health under the reference BB/H020195/1.

\section{Availability of data and material}

Not applicable.

\section{Authors' contributions}

VMH, FMT and DPB conceived and designed the experiments. VMH, SM, EP and $\mathrm{ChC}$ generated the transgenic parasites. VMH characterized the transgenic populations. CC coordinated the immunological experiments. $\mathrm{VMH}$ and $\mathrm{ChC}$ performed the lymphoproliferation assays and Western blots. $\mathrm{VMH}$ analyzed the data and wrote the manuscript. DPB, FMT and CC critically reviewed and modified the paper. All authors read and approved the final version of the manuscript.

\section{Competing interests}

The authors declare that they have no competing interests.

\section{Consent for publication}

Not applicable.

\section{Ethics approval and consent to participate}

This study was carried out in strict accordance with the Animals (Scientific Procedures) Act 1986, an Act of Parliament of the United Kingdom. All animal studies and protocols were approved by the Royal Veterinary College and/or MSD Animal Health Ethical Review Committees and the United Kingdom Government Home Office under specific project licence. The laboratory work involving GMO was conducted under authorization GM9708.1, administered by the UK Health and Safety Executive. The animal facilities for GMO are classified derogated containment level 3.

\section{Author details}

${ }^{1}$ The Royal Veterinary College, University of London, Hawkshead Lane, North Mymms AL9 7TA, UK. 'MSD Animal Health, Walton Manor, Milton Keynes MK7 7AJ, UK.

\section{Received: 31 May 2016 Accepted: 12 August 2016}

Published online: 23 August 2016

\section{References}

1. Food and Agriculture Organization of the United Nations Statistics Division. http://faostat3.fao.org. Accessed 8 Apr 2016

2. Haslam S. Legislation and Poultry Management, Poultry Diseases Sixth Edition Saunders Elsevier. 2008.

3. Dhama K, Rajagunalan S, Chakraborty S, Verma AK, Kumar A, Tiwari R, Kapoor S. Food-borne pathogens of animal origin-diagnosis, prevention, control and their zoonotic significance: a review. Pak J Biol Sci. 2013;16(20):1076-85

4. Lister S. Biosecurity and Poultry Welfare, Poultry Diseases Sixth Edition Saunders Elsevier. 2008.

5. Cserep T. Vaccines and Vaccination, Poultry Diseases Sixth Edition Saunders Elsevier. 2008.

6. Vaccination Programs in Poultry. http://www.merckvetmanual.com/mvm/ index.html. Accessed 8 Apr 2016.
7. Chapman HD, Jeffers TK. Vaccination of chickens against coccidiosis ameliorates drug resistance in commercial poultry production. Int J Parasitol Drugs and Drug Resist. 2014;4(3):214-7.

8. Blake DP, Tomley FM. Securing poultry production from the ever-present Eimeria challenge. Trends Parasitol. 2014;30(1):12-9.

9. Clark JD, Billington K, Bumstead JM, Oakes RD, Soon PE, Sopp P, Tomley FM, Blake DP. A toolbox facilitating stable transfection of Eimeria species. Mol Biochem Parasitol. 2008;162(1):77-86.

10. Clark JD, Oakes RD, Redhead K, Crouch CF, Francis MJ, Tomley FM, Blake DP. Eimeria species parasites as novel vaccine delivery vectors: antiCampylobacter jejuni protective immunity induced by Eimeria tenelladelivered CjaA. Vaccine. 2012;30(16):2683-8.

11. Liu X, Zou J, Yin G, Su H, Huang X, Li J, Xie L, Cao Y, Cui Y, Suo X Development of transgenic lines of Eimeria tenella expressing M2e-enhanced yellow fluorescent protein (M2e-EYFP). Vet Parasitol. 2013;193(1-3):1-7.

12. Yun CH, Lillehoj HS, Choi KD. Eimeria tenella infection induces local gamma interferon production and intestinal lymphocyte subpopulation changes. Infect Immun. 2000;68(3):1282-8.

13. Muller $\mathrm{H}$, Mundt $\mathrm{E}$, Eterradossi $\mathrm{N}$, Islam MR. Current status of vaccines against infectious bursal disease. Avian Pathol. 2012;41(2):133-9.

14. Maity HK, Dey S, Mohan CM, Khulape SA, Pathak DC, Vakharia VN. Protective efficacy of a DNA vaccine construct encoding the VP2 gene of infectious bursal disease and a truncated HSP70 of Mycobacterium tuberculosis in chickens. Vaccine. 2015;33(8):1033-9.

15. Taghavian O, Spiegel H, Hauck R, Hafez HM, Fischer R, Schillberg S. Protective oral vaccination against infectious bursal disease virus using the major viral antigenic protein VP2 produced in Pichia pastoris. PLoS One. 2013:8(12):e83210

16. Rong J, Jiang T, Cheng T, Shen M, Du Y, Li S, Wang S, Xu B, Fan G. Large-scale manufacture and use of recombinant VP2 vaccine against infectious bursal disease in chickens. Vaccine. 2007;25(46):7900-8.

17. Ou SC, Giambrone JJ. Infectious laryngotracheitis virus in chickens. World J Virol. 2012;1(5):142-9.

18. McDougald LR, Jeffers TK. Comparative in vitro development of precocious and normal strains of Eimeria tenella (Coccidia). J Protozool. 1976;23(4):530-4.

19. Long $\mathrm{PL}$, Millard BJ, Joyner LP, Norton CC. A guide to laboratory techniques used in the study and diagnosis of avian coccidiosis. Folia Vet Lat. 1976:6(3):201-17.

20. Shirley M. Eimeria species and strains of chickens. In: Eckert J, Braun R, Shirley M, Coudert P, editors. Guidelines on techniques in coccidiosis research. Luxembourg: European Commission; 1995

21. Kelleher M, Tomley FM. Transient expression of beta-galactosidase in differentiating sporozoites of Eimeria tenella. Mol Biochem Parasitol. 1998;97(1-2):21-31.

22. Liu X, Shi T, Ren H, Su H, Yan W, Suo X. Restriction enzyme-mediated transfection improved transfection efficiency in vitro in Apicomplexan parasite Eimeria tenella. Mol Biochem Parasitol. 2008;161(1):72-5.

23. Huang X, Zou J, Xu H, Ding Y, Yin G, Liu X, Suo X. Transgenic Eimeria tenella expressing enhanced yellow fluorescent protein targeted to different cellular compartments stimulated dichotomic immune responses in chickens.J Immunol. 2011:187(7):3595-602.

24. Pavlova S, Veits J, Mettenleiter TC, Fuchs W. Identification and functional analysis of membrane proteins $\mathrm{gD}, \mathrm{gE}, \mathrm{gl}$, and pUS9 of Infectious laryngotracheitis virus. Avian Dis. 2013;57(2 Suppl):416-26.

25. Walker RA, Sharman PA, Miller CM, Lippuner C, Okoniewski M, Eichenberger RM, Ramakrishnan C, Brossier F, Deplazes P, Hehl AB, et al. RNA Seq analysis of the Eimeria tenella gametocyte transcriptome reveals clues about the molecular basis for sexual reproduction and oocyst biogenesis. BMC Genomics. 2015;16:94

26. Li L, Fang W, Li J, Huang Y, Yu L. Oral DNA vaccination with the polyprotein gene of infectious bursal disease virus (IBDV) delivered by the attenuated Salmonella elicits protective immune responses in chickens. Vaccine. 2006;24(33-34):5919-27.

27. Oin M, Liu XY, Tang XM, Suo JX, Tao GR, Suo X. Transfection of Eimeria mitis with yellow fluorescent protein as reporter and the endogenous development of the transgenic parasite. PLoS One. 2014:9(12):e114188.

28. Yan W, Liu X, Shi T, Hao L, Tomley FM, Suo X. Stable transfection of Eimeria tenella: constitutive expression of the YFP-YFP molecule throughout the life cycle. Int J Parasitol. 2009;39(1):109-17.

29. Reid AJ, Blake DP, Ansari HR, Billington K, Browne HP, Bryant J, Dunn M, Hung SS, Kawahara F, Miranda-Saavedra D, et al. Genomic analysis of the 
causative agents of coccidiosis in domestic chickens. Genome Res 2014;24(10):1676-85.

30. Pradhan SN, Prince PR, Madhumathi J, Roy P, Narayanan RB, Antony U. Protective immune responses of recombinant VP2 subunit antigen of infectious bursal disease virus in chickens. Vet Immunol Immunopathol. 2012;148(3-4):293-301.

31. Xu XG, Tong DW, Wang ZS, Zhang Q, Li ZC, Zhang K, Li W, Liu HJ. Baculovirus virions displaying infectious bursal disease virus VP2 protein protect chickens against infectious bursal disease virus infection. Avian Dis. 2011;55(2):223-9.

32. Truong $A D$, Hong $\mathrm{YH}$, Lillehoj $\mathrm{HS}$. High-throughput sequencing reveals differing immune responses in the intestinal mucosa of two inbred lines afflicted with necrotic enteritis. Vet Immunol Immunopathol. 2015;166(3-4):116-24.

33. Pradhan SN, Prince PR, Madhumathi J, Arunkumar C, Roy P, Narayanan RB, Antony U. DNA vaccination with VP2 gene fragment confers protection against Infectious Bursal Disease Virus in chickens. Vet Microbiol. 2014:171(1-2):13-22.

34. Gregg B, Dzierszinski F, Tait E, Jordan KA, Hunter CA, Roos DS. Subcellular antigen location influences T-cell activation during acute infection with Toxoplasma gondii. PLoS One. 2011;6(7):e22936.

35. Sidik SM, Hackett CG, Tran F, Westwood NJ, Lourido S. Efficient genome engineering of Toxoplasma gondii using CRISPR/Cas9. PLoS One. 2014;9(6):e100450.

36. Shen B, Brown KM, Lee TD, Sibley LD. Efficient gene disruption in diverse strains of Toxoplasma gondii using CRISPR/CAS9. mBio. 2014;5(3):e01114-01114.

\section{Submit your next manuscript to BioMed Central} and we will help you at every step:

- We accept pre-submission inquiries

- Our selector tool helps you to find the most relevant journal

- We provide round the clock customer support

- Convenient online submission

- Thorough peer review

- Inclusion in PubMed and all major indexing services

- Maximum visibility for your research

Submit your manuscript at www.biomedcentral.com/submit

) Biomed Central 